\title{
Multiobjective VAR planning using the goal-attainment method
}

\author{
Y.-L. Chen \\ C.-C. Liu
}

\begin{abstract}
In the paper, a new formulation of multiobjective optimisation (MO) for reactive power (VAR) planning is presented. The objectives are active power loss cost reduction, minimisation of the cost of the investment VAR sources, system security margin robustness and reduction of the voltage deviation of the system. The operating constraints, load constraints of the system and expansion constraints, are taking into consideration. Hence, the new formulation is a constrained, multiobjective and nondifferentiable optimisation problem with both equality and inequality constraints. The goal-attainment method, based on the simulated annealing (SA) approach to solving general multiobjective optimisation problems by assuming that the decision maker (DM) has goals for each of the objective functions, is presented. The solution methodology can find a desirable, global noninferior solution for the MO problem, even when the objective space is nonconvex. Results of application of the method to multiobjective VAR planning are also presented.
\end{abstract}

\section{Introduction}

The goal of VAR planning is to provide the system with enough VAR sources for the system to operate in an economic condition with enhanced system security, while load constraints and operational constraints with respect to credible contingencies are met.

In the past [1-10], many formulations for a more systematic approach to VAR planning have been developed. Many of these formulations are based on linearprogramming methods, and the objective function is treated as continuous [3,4]. Sachdeva and Billinton [5] proposed a nonlinear analysis scheme for the planning of a network compensator. An efficient dual simplex linearprogramming technique coupled with relaxation and contingency analysis was employed for problem solving [8]. Obadina and Berg [9] presented a method for identifying dispersed reactive power supplies that would enhance power systems' pre- and post-contingency voltage security, subject to technical and economic constraints. A common characteristic of these methods was that the value of VAR sources was still treated as contin-

(ㄷ) IEE, 1994

Paper $9941 \mathrm{C}$ (P9), first received 3rd June and in revised form 4th October 1993

The authors are with the Department of Electrical Engineering, National Taiwan University, Taipei, Taiwan, Republic of China

IEE Proc.-Gener. Transm. Distrib., Vol. 141, No. 3, May 1994 uously differentiable, and they expressed the multiobjective VAR planning problem as a single-objective optimisation. The salient feature was that the system security margin was not taken into consideration in the problem $[1-8,10]$.

This paper presents a new formulation of multiobjective optimisation for VAR planning. The objectives consist of three important terms, which are the economic operation condition of system, the system security margin and the voltage deviation of the system. The operating constraints, load constraints and the new VAR source expansion constraints are taken into consideration.

In multiobjective problems, the objectives are usually noncommensurable and usually conflict with each other in that any improvement of one objective can be reached only at the loss of another. It is necessary to present a DM for the problem that implies the trade-offs between objectives. The aim of this problem is to find a compromise for the satisfying solution of the DM. It can be readily handled by the goal-attainment $(\mathrm{GA})$ method. The SA technique is a powerful tool for solving the VAR planning problem, which belongs to a class of com. binatorial optimisation problems, and it can search for the global optimal solution [15-22]. As a result, the solution methodology presented, the GA method based on the SA technique, can find a desirable, global noninferior solution for the multiobjective optimal VAR planning problem.

In this paper, the contributions are summarised as follows: A new formulation of MO for VAR planning is presented. Not only is economic operation taken into consideration, but the system security margin is taken into account. The new formulation represents a more realistic mathematical model for the actual behaviour of a power system. The GA method based on SA is presented to solve for a general MO problem by assuming that the DM has goals for each objective, and it is not subject to convexity limitations of any kind. The solution methodology can handle easily a multiobjective, nondifferentiable optimisation problem with both equality and inequality constraints. The solution methodology can search for a desirable, global noninferior solution for a general MO problem, even if a nonconvex objective space is met.

\section{Problem formulation}

In this Section, a new formulation for multiobjective VAR planning is presented. This formulation treats the problem as a multiobjective mathematical programming problem, which is concerned with the attempt to minimise each objective, simultaneously. Meanwhile, the equality and inequality constraints of the system must be satisfied. 


\subsection{Load constraints}

The load constraints are real and reactive power balance load flow equations, which hold for every busbar of the system. These equality constraints can be written in compact form as

$$
L(x, u, w)=0
$$

where $x$ and $u$ imply the state variable vector and the control variable vector, respectively, and $w$ is the vector of VAR expansion variables.

\subsection{Operating constraints}

The system operating constraints consist of the available range of active and reactive generated power, the bound of the controlled transformer tap change, line flow limits, security bounds on voltage magnitudes, and limits on voltage angle difference between every two busbars. These constraints are summarised in a compact form as

$$
G(x, u, w) \leqslant 0
$$

\subsection{Expansion constraints}

The expansion constraints are the limits of the installation of new VAR sources. For instance, it is impossible to install more VAR sources if there is not enough space in a substation, so that to install the sizes and types of the VAR sources needed also lies on their space upper limits. We express the vector of expansion variables $w$, constrained by

$$
\left\{\begin{array}{l}
0 \leqslant q_{r i} \leqslant q_{r i}^{\max } \\
0 \leqslant q_{c i} \leqslant q_{c i}^{\max }
\end{array} \quad i \in \Omega_{L}\right.
$$

where $q_{c i}$ and $q_{r i}$ are the added capacitive and inductive compensations at busbar $i$, respectively. They are all integer numbers, and $\Omega_{L}$ is a set of all candidate busbars at which new VAR sources can be installed. For convenience, we express the above set of constraints in compact form as

$$
R(w) \leqslant 0
$$

\subsection{Objective functions}

The objective functions of the reactive power planning problem comprise three important terms, which are the system loss and VAR source investment costs, the system security margin and the voltage deviation of the system.

2.4.1 Loss and investment costs: This objective is to minimise the total real power loss cost and the purchase cost of the newly installed VAR sources, which can be described as

$$
f_{1}(x, u, w)=K_{\mathrm{e}} P_{\text {loss }}(x, u, w) D_{i}+C(w)
$$

where $K_{e}(\$ / \mathrm{kWh})$ is the cost of real power, $D_{i}$ is the duration of the system operating time, $P_{\text {loss }}(x, u, w)$ is the system real power loss, and $C(w)$ is the total purchase cost of the newly installed VAR sources, illustrated as

$$
C(w)=\sum_{i \in \Omega_{l}}\left(d_{i}+s_{c i} q_{c i}+s_{r i} q_{r i}\right)
$$

where $d_{i}$ is the installment cost at busbar i. $s_{c i}$ and $s_{r i}$ are the unit costs of the capacitor and reactor, respectively. $w$ is called the expansion variable vector, and it indicates whether or not to install VAR sources and identifies the VAR source types and sizes.

2.4.2 System security margin: This objective is concerned with the static voltage stability of the system and investigates how to reduce the risk of voltage collapse in the system. Voltage collapse means that the system is unable to meet a given load demand, and this situation is a critical system state. Knowing the critical state, an indication of the system security from voltage collapse is available. Having determined the critical state, a security margin $S M$ can be defined as

$$
S M=\frac{\sum_{j \in J_{L}} S_{j}^{l i m i t}-\sum_{j \in J_{L}} S_{j}^{\text {initial }}}{\sum_{j \in J_{L}} S_{j}^{\text {limit }}}
$$

where $S_{j}^{\text {initial }}$ and $S_{j}^{\text {limit }}$ are the MVA loads of busbar $j$ at initial state and critical state, respectively. $J_{L}$ is a set of load busbars. For a stable system operating condition, $S M$ must take values between 0 and 1 . A negative value of $S M$ means that the system is unable to supply the specified initial MVA demand, and the system is in voltage collapse. In the VAR planning problem, to reduce the risk of voltage collapse of the system means maximising $S M$ by installing VAR sources or not. To match the minimisation problem, this objective is defined as

$$
f_{2}(x, u, w)=1-S M=\frac{\sum_{j \in J_{L}} S_{j}^{\text {initial }}}{\sum_{j \in J_{L}} S_{j}^{\text {limir }}}
$$

To minimise this objective involves keeping away from the voltage circuit critical (collapse) state, and then the system can suffer more severe contingencies (e.g. line outage etc.) but not voltage collapse. $(1-S M)=1$ at the voltage critical state. $(1-S M)>1$ at the voltage collapse state, and $(1-S M)<1$ at the voltage stable state.

The following nonlinear optimisation problem is solved to determine the critical MVA load demand $\sum_{j \in J_{L}} S_{j}^{\text {limit }}$ [11], and we briefly discuss it as below.

Maximise (total MVA demand) subject to

(a) distribution constraints at load busbars

(b) MVAR and MW limits on generators

(c) generator MW participation

(d) load-voltage characteristics at load busbars

(e) constant power factor of MVA demand aps.

$(f)$ limits on controlled voltages and LTC transformer

The above constraints may be briefly summarised as follows (see Reference 11 for more detail)

(a) These constraints describe and enforce a prescribed pattern of increase of the MVA demand vector.

(b) These contrain the MW and MVAR outputs of the respective generators to be within their specified limits.

(c) As system demand increases, each generating-unit MW output needs to be changed to participate in the load change. This constraint set enforces a prescribed participation of system active power production

(d) The static load-voltage characteristics are enforced by this constraint set. The voltage dependence of the load at busbar $i$ is modelled as

$$
\begin{aligned}
P_{L i} & =P_{o i} V_{i}^{p_{i}} \\
Q_{L i} & =Q_{o i} V_{i}^{q_{i}}
\end{aligned}
$$

where $P_{o i}$ and $Q_{o i}$ are the prescribed real and reactive loads at rated (normal) voltage, and $p_{i}$ and $q_{i}$ are constants that reflect the load-voltage characteristics at busbar $i$.

(e) This is a constraint set that maintains a specified power factor for the respective busbar loads

(f) Controlled voltages are maintained within specified limits if the generator AVR or the LTC is still operative.

IEE Proc-Gener. Transm Distrib., Vol 14I, No. 3, May 1994 
2.4.3 Voltage-magnitude deviation: This objective is concerned with the voltage deviation of the system. Voltage deviation in each busbar is defined as

$$
V D_{i}=\frac{\Phi\left(\left|V_{i}-V_{i}^{\text {ideal }}\right|-d v_{i}\right)}{V_{i}}
$$

where

$$
\Phi(x)= \begin{cases}0 & \text { if } x<0 \\ x & \text { otherwise }\end{cases}
$$

and $V_{i}$ is a voltage magnitude at busbar $i, V_{i}^{i d e a l}$ is the ideal specific voltage at busbar $i$ and is usually set to be 1 p.u., and $d v_{i}$ is the tolerance of maximum deviation in the voltage. It is obvious that a voltage between its crisp limits will have a value of zero (i.e. $V D_{i}=0$ ). As the voltage exceeds this limit, the value of $V D_{i}$ increases and depends on the operating conditions of the system. Therefore the objective of the voltage profile is considered as follows

$$
f_{3}(x, u, w)=\sum_{i \in J_{L}} V D_{i}=\sum_{i \in J_{L}} \frac{\Phi\left(\left|V_{i}-V_{i}^{\text {ideat }}\right|-d v_{i}\right)}{V_{i}}
$$

To minimise this objective means trying to push the voltage values towards its crisp limits.

\subsection{Overall problem}

We discuss the issue of co-ordinating the above three objective functions namely

(a) minimisation of the operating and investment costs

(b) enhanced system security margin

(c) minimisation of voltage deviation

In summary, we want to seek an optimal VAR planning configuration $\chi^{*}$ among all possible configurations $\chi$ by installing VAR sources or not, such that all objective functions are optimised while the load constraints, operating constraints and expansion constraints are satisfied. The mathematical formulation for this problem is expressed as

$$
\begin{aligned}
& \min _{u, w \in \chi} f_{1}(x, u, w) \\
& \min _{u, w \in \chi} f_{2}(x, u, w) \\
& \min _{u, w \in x} f_{3}(x, u, w)
\end{aligned}
$$

subject to (1)-(3).

As a result, the VAR planning problem is a constrained, multiobjective, nondifferentiable optimisation problem. The goal-attainment method based on the SA approach for solving this problem will be presented in this paper.

Before leaving this Section, we must define a specific and very important concept of the noninferior set in the general MO problem [12].

Definition (noninferior solution): $z^{*}$ is said to be a noninferior solution of $\mathrm{MO}$ if there exists no other feasible (i.e. $z \in \chi)$ such that $f(z) \leqslant f\left(z^{*}\right)$, meaning $f_{i}(z) \leqslant f_{\mathcal{A}}\left(z^{*}\right)$ for all $i=1,2, \ldots, m$, with strict inequality for at least one $i$ $f\left(z^{*}\right)$ is said to be a noninferior solution of $\mathrm{MO}$ in objective space.

\section{The goal-attainment method}

The goal-attainment (GA) method $[13,14]$ is a power tool to find the best-compromise solution in MO prob-

IEE Proc.-Gener. Transm. Distrib., Vol. 141, No. 3, May 1994 lems and it is not subject to convexity limitations of any kind. In this approach, a vector of weights $w$ relating the relative under- or over-attainment of the desired goals must be elicited from the decision maker in addition to the goal vector $y$. To find the best-compromise solution, we solve the following problem

$$
\min _{z \in X} \alpha
$$

subject to

$$
\boldsymbol{y}+\alpha \boldsymbol{w} \geqslant f(z) \quad w \in \Lambda_{\varepsilon}
$$

where $\alpha$ is a scalar variable unrestricted in sign, $\chi$ is a feasible-solution region, and $\Lambda_{c}=\left\{w \in \mathscr{R}^{m}\right.$ s.t. $w_{i} \geqslant \varepsilon$, $\sum_{i=1}^{m} w_{i}=1$ and $\left.\varepsilon \geqslant 0\right\}$. If some $w_{i}=0$ (components of vector $w$ ), it means that the maximum limit of objectives $f_{i}(z)$ is $y_{i}$.

It can be easily shown that the set of noninferior solutions can be generated by varying $w$ over $\Lambda_{\varepsilon}$ with $\varepsilon=0$ even for nonconvex problems. The mechanism by which this method operates is illustrated for the two-objective case in Fig. 1. The vector $y$ is represented by the decision

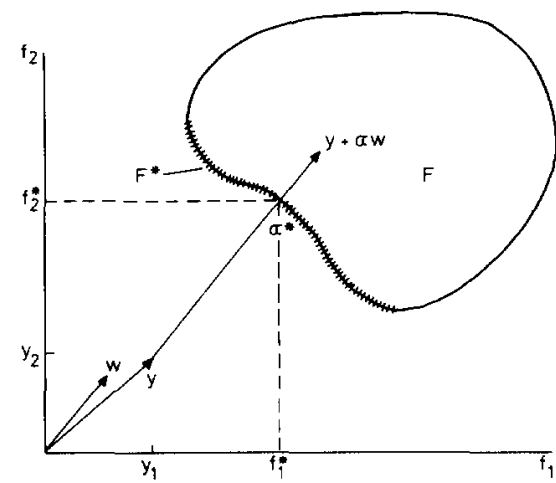

Fig. 1 Goal-attainment method with two-objective case

goal of the DM, and the direction of $w$ is the preference direction of the DM. Given vector $w$ and $y$, the direction of the vector $y+\alpha w$ can be determined, and the problem eqn. 12 is equivalent to finding a feasible point on this vector in objective space which is closest to the origin. It is obvious that the optimal solution of eqn. 12 will be the first point at which $y+\alpha w$ intersects the feasible region $F$ in the objective space. Should this point of intersection exist, it would clearly be a noninferior solution.

It should be pointed out that the optimum value of $\alpha$ will inform the DM of whether the goals are attainable or not. A negative value of $\alpha$ implies that the goal of the DM is attainable, and an improved solution will be obtained. Otherwise, if $\alpha>0$, then the DM goal is unattainable.

\section{Simulated annealing}

Simulated annealing is a powerful general-purpose technique for solving combinatorial optimisation problems. It is a randomisation algorithm and can asymptotically search for a global optimum solution with probability of 1 [15]. This technique exploits the resemblance between a minimisation process and the cooling of molten metal. The energy of the metal is minimal when the annealing process is finished. We will obtain an equivalence: the cost is minimal as the SA technique is applied. In this 
algorithm, a parameter $T$ called temperature is defined. The algorithm of SA can be described as below.

Simulated annealing algorithm:

Step 1: Randomly choose an initial condition (solution)

Step 2: Generate a feasible point in the neighbourhood of the current point from solution space

Step 3: Evaluate the increase in the cost $\Delta C$

Step $4 ;:$ If $\Delta C<0$, then accept the new solution point and go to Step 6

Step 5: A random number $r$ uniformly distributed in the interval $[0,1)$ is chosen. If $\exp (-\Delta C / T)>r$, then accept the new point, otherwise, the new point is discarded.

Step 6: If the moves are not finished, then go to Step 2

Step 7: Cooling down temperature $T=\beta * T$

Step 8: If $T>T_{\min }$, go to Step 2

Step 9: Output global optimal solution

At each temperature, randomly choose a new solution point $S^{\prime}$ by perturbing the current point $S$. Then, evaluate the increase cost, $\Delta C=\operatorname{cost}\left(S^{\prime}\right)-\operatorname{cost}(S)$. If the move that reduces the cost is downhill, then the move is accepted. If the move that increases the cost is uphill, then the move is accepted with a probability of $\exp (-\Delta C / T)$. Owing to the probabilistic selection rule, SA can always escape out of a local optimum in which it could become trapped and proceed to the desired global optimum. This feature distinguishes the SA from the greedy search approach $[15,16,19]$.

The temperature is lowered according to the rules $T_{k+1}=\beta\left(T_{k}\right) * T_{k}$, where $\beta\left(T_{k}\right)$ is a constant smaller than, but close to, 1 . Typical values lie between 0.8 and 0.99 . When the acceptance ratio is low or the sample mean and variance of cost values at current temperature have big drops, then $\beta\left(T_{k}\right)$ is adjusted to a higher value to avoid becoming stuck at a local optimum point. Otherwise, $\beta\left(T_{k}\right)$ is adjusted to a lower value (say 0.85 ) to increase the convergence speed.

The advantages of the SA approach applied to optimum VAR source planning are that it can handle a mixed-integer nonlinear-programming problem and can search towards a (near) global optimum solution [20 22]. Hence, the GA method based on SA for multiobjective VAR planning is presented in the following Section.

\section{Solution algorithm}

A best-compromise, (near) global optimum solution will be obtained by the GA method based on SA methodology. An MO problem can be converted into a singleobjective optimisation by the GA method, and a single-objective optimisation can easily be handled by the SA approach. The solution algorithm proposed can offer the global optimum solution for the multiobjective VAR planning problem and is described as below.

Solution algorithm for multiobjective VAR source planning:

* Initialisation */

Step la: Input system data, vector $w$ and the decision vector $\boldsymbol{y}$. The direction of vector $\boldsymbol{w}$ is the direction of preference of the DM to approach the noninferior solution. The decision vector $y$ is given by the DM (then go to Step 2) or automatically generated by Step $1 b$.
Step $1 b$ : To give a decision point automatically. Using a single-objective optimisation method to determine every decision component of objective functions

$$
y_{i}=\min _{z \in \chi} f_{i}(z) \quad \text { for } i=1,2, \ldots, m
$$

* The GA method based on SA */

Step 2: Input control parameters. Input control parameters, such as the initial temperature, random number seed, cooling rate, the step size of control setting (e.g. 3 MVAR) and the number of moves at each temperature.

Step 3: Obtain an initial feasible configuration.

(1) Generate a new configuration using a perturbation mechanism (see References 20-22 for more detail)

(2) For the new configuration, run a load flow to check the feasibility. If any constraint is violated, go to (1). Otherwise, proceed to (3)

(3) Calculate each objective function $f_{i}(z)$

(4) Check the objective constraints if some $w_{i}=0$

If $w_{i}=0$ and $f_{i}(z)>y_{i}$, go to (1); otherwise, proceed to (5)

(5) Search the value of $\alpha$ using the following criterion

$$
\alpha=\max _{i} \frac{f_{i}(z)-y_{i}}{w_{i}} \text { for all } w_{i} \neq 0
$$

Step 4: Design a proper global cooling schedule. At each temperature $T_{k}$, perform a number of $L_{k}$ moves. For move $=1,2, \ldots, L_{k}$, do steps 5-7. Otherwise, proceed to Step 8.

Step 5: Generate a new feasible configuration.

(1) Generate a new configuration using a perturbation mechanism.

(2) For the new configuration, run a load flow to check the feasibility. If any constraint is violated, go to (1). Otherwise, proceed to (3).

(3) Calculate each objective function $f_{i}(z)$.

(4) Check the objective constraints if some $w_{i}=0$.

(5) Search for the value of $\alpha$ using the criterion of eqn.

Step 6: Update the system configuration. Use the value of $\Delta \alpha$ to determine the system configuration. Retain the new configuration or restore it to the previous configuration based on the acceptation criterion.

Acceptation probability

$$
\begin{aligned}
& P_{c}\{\text { accept new configuration }\} \\
& = \begin{cases}1 & \text { if } \Delta \alpha \leqslant 0 \\
\exp \left(\frac{-\Delta \alpha}{T_{k}}\right) & \text { otherwise }\end{cases}
\end{aligned}
$$

Step 7: Check the stop criterion. If the stop criterion is not satisfied, then the system is not yet frozen; continue the process by returning to Step 4 . Otherwise, proceed to next step.

Step 8: Print out the global optimum configuration.

In the above algorithm, eqn. 14 is a function that forces the new configuration to satisfy the constrain $y+\alpha w \geqslant f(z)$ in the GA method by choosing the largest value $\alpha$, and the SA approach is applied to minimise the value $\alpha$. It is obvious that Step $1 b$ can be automatically given an initial decision preference $y$ for the DM. The direction of vector $w$ is chosen for the DM preference direction from point $y$ to close a noninferior solution in objective space. Given vector $\boldsymbol{w}$ and $\boldsymbol{y}$, the methodology 
of the GA method based on the SA approach can find a feasible point on vector $y+\alpha w$ in objective space, the solution point is that closest to the origin, and this feasible point is a noninferior solution of the problem.

The output of the above solution algorithm gives the optimum configuration of the installed VAR sources, and the solution point is matched to the decision point along the direction $w$ as closely as possible. A numerical study with very promising results is addressed in the following Section.

\section{Numerical examples}

The results of the application of the proposed method to the AEP 14-busbar system [11] are now presented. The initial operating conditions are shown in Table 1 and 2.

Table 1: Estimate of initial operating condition (AEP 14 busbar)

\begin{tabular}{|c|c|c|c|c|c|}
\hline \multirow[t]{2}{*}{ Busbar } & \multicolumn{2}{|c|}{$\begin{array}{l}\text { Busbar } \\
\text { voltage }\end{array}$} & \multicolumn{2}{|c|}{$\begin{array}{l}\text { Busbar } \\
\text { power* }\end{array}$} & \multirow[t]{2}{*}{$\begin{array}{l}\text { Statict } \\
\text { shunt }\end{array}$} \\
\hline & magnitude & angle $N$ & MW & MVAR & \\
\hline 1 & 1.06 & 0.0 & - & - & \\
\hline 2 & 1.045 & 0.0 & 40.0 & 0.0 & \\
\hline 3 & 1.01 & 0.0 & -94.2 & -19.0 & \\
\hline 4 & 1.0 & 0.0 & -57.8 & -23.9 & \\
\hline 5 & 1.0 & 0.0 & -47.6 & -1.6 & \\
\hline 6 & 1.01 & 0.0 & 0.0 & 0.0 & \\
\hline 7 & 1.0 & 0.0 & 0.0 & 0.0 & \\
\hline 8 & 1.01 & 0.0 & 0.0 & 0.0 & \\
\hline 9 & 1.0 & 0.0 & -29.5 & -16.6 & 0.19 \\
\hline 10 & 1.0 & 0.0 & -29.5 & -5.8 & \\
\hline 11 & 1.0 & 0.0 & -13.5 & -5.8 & \\
\hline 12 & 1.0 & 0.0 & -36.1 & -11.6 & \\
\hline 13 & 1.0 & 0.0 & -23.5 & -15.8 & \\
\hline 14 & 1.0 & 0.0 & -14.9 & -10.0 & \\
\hline \multicolumn{6}{|c|}{$\begin{array}{l}* \text { for load } \\
\text { t } 100 \text { MVAR base }\end{array}$} \\
\hline \multirow[t]{2}{*}{ Busbar } & \multicolumn{2}{|c|}{ MVAR limits } & \multicolumn{3}{|c|}{ MW limits } \\
\hline & minimum & maximum & $m \quad \min$ & imum & maximum \\
\hline 2 & -6.0 & 24.0 & 30.0 & & 70.0 \\
\hline 3 & -6.0 & 24.0 & 0.0 & & 0.0 \\
\hline 6 & -6.0 & 24.0 & $0 . c$ & & 0.0 \\
\hline 8 & -6.0 & 24.0 & 0.0 & & 0.0 \\
\hline
\end{tabular}

The constant power model is applied to all load demands. To determine the maximum MVA load demand, $\sum_{j \in J_{L}} S_{j}^{\text {limit }}$, the components of the distribution vector $\rho$ are chosen to be $\rho_{i}=S_{i}^{\text {initial }} / \sum_{j \in J_{t}} S_{j}^{\text {initial }}$, and the participation factors of the generating unit are chosen as in Reference 11.

Two different studies illustrate the performance of the solution algorithm proposed. For all studies, one bank of the VAR source is set to be 3 MVAR, the specific voltage $V_{i}^{\text {ideal }}$ of all load busbars is set to be 1 p.u., $d v_{i}$ is set to be 0.02 p.u. (the crisp limits of voltage in each load busbar are between $0.98-1.02$ p.u.), and the following parameters are used: power loss cost weight $\left(K_{e}=2.31 \$ \mathrm{NT} / \mathrm{kWh}\right.$, $D_{i}=10 \times 365 \times 24(\mathrm{~h})$, ten years), VAR source cost weight $\quad\left(s_{c i}=11385 \$ \mathrm{NT} / \mathrm{bank}, \quad s_{r \mathrm{i}}=16400 \$ \mathrm{NT} / \mathrm{bank}\right.$ and $d_{i}=420000 \$ N T /$ location), and $q_{c i}^{\max }$ and $q_{r i}^{\max }$ are all limited to 90 MVAR (30 banks). The $K$ parameter is the average cost of real power in Taiwan currently. The lifetime of the VAR compensator is determined as ten years, and the other cost parameters are all estimated according to the real conditions in Taiwan.

IEE Proc.-Gener. Transm. Distrib., Vol. 141, No. 3, May 1994
In study 1 , busbars $4,5,9,11,13$ and 14 are selected as candidate busbars for the installation of new VAR sources (i.e. $\Omega_{L}=\{4,5,9,11,13,14\}$ ). At the initialisation process, a decision vector $\boldsymbol{y}=\left[y_{1}, y_{2}, y_{3}\right]^{T}=[0.5,0.6$, $0.0]^{T}$ and vector $w=\left[w_{1}, w_{2}, w_{3}\right]^{T}=[0.4,0.3,0.3]^{T}$ are given by the DM. $y_{1}$ is the operation and investment cost objective, which has been expressed by $10^{10}$ \$NT base, $y_{2}$ is the system security margin objective, and $y_{3}$ is the voltage deviation objective. The very promising results are presented in Table 3 . In this study, the final result can save \$NT 1618 million.

Table 3: Result of study 1 (AEP 14-busbar system)

\begin{tabular}{lllll}
\hline & $\begin{array}{l}\text { Before } \\
\text { planning }\end{array}$ & $\begin{array}{l}\text { DM } \\
\text { goals }\end{array}$ & $\begin{array}{l}\text { After } \\
\text { planning }\end{array}$ & $\begin{array}{l}\% \\
\text { reduction }\end{array}$ \\
\hline Loss $(100 \mathrm{MW})$ & 0.36799 & - & 0.2879 & 21.75 \\
Cost $\left(\times 10^{10}\right.$ \$NT $)$ & 0.74465 & 0.500 & 0.5829 & 21.71 \\
$1-S M$ & 0.90539 & 0.600 & 0.6714 & 22.50 \\
$\sum_{\alpha} V D ;$ & 1.19330 & 0.000 & 0.0725 & 93.92 \\
& 3.97760 & - & 0.2418 & - \\
\hline$w=\left[\begin{array}{lllll}0.4 & 0.3 & 0.3\end{array}\right]^{T}$ & & & &
\end{tabular}

The convergence behaviours of the proposed method in study 1 are shown in Figs. 2 and 3. Fig. 2 shows the value of $\alpha$ plotted as a function of the temperature. The

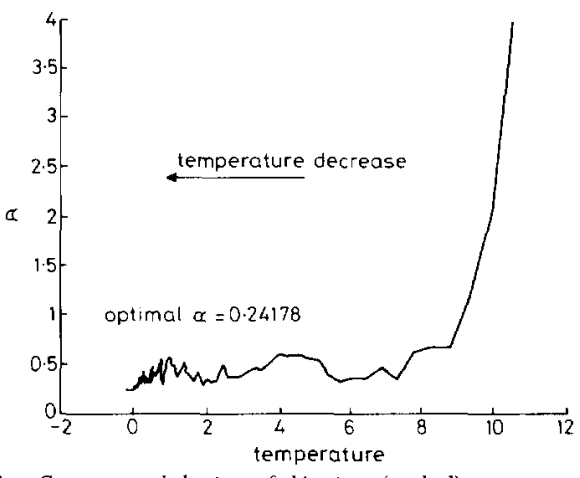

Fig. 2 Convergence behaviour of objective $\alpha$ (study 1$)$

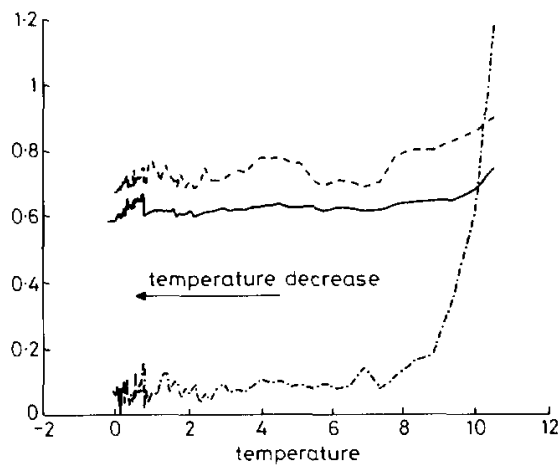

Fig. 3 Convergence trajectories of objectives (study 1 ) Cost $\left(\times 10^{10}\right.$ \$NT $)$ $1-S M)$

Voltage deviation

optimal value $\alpha$, which is a positive number, informs the DM that the decision strategy is unattainable. It also should be pointed out that a zigzag convergence trajectory implies that the SA approach escapes from a local 
minimum towards a global minumum. The convergence trajectories of the objectives are shown in Fig. 3. This study required $866 \mathrm{~s}$ total computer time on a SUN SPARC Station 2 to obtain the optimum solutions. In spite of the solution algorithm is wasteful time to obtain optimal solution, but it is not important to apply in the planning problem, because the optimal solution is more attractive than the computation time in the planning problems.

In study 2, all load busbars are selected as candidate busbar for the installation of new VAR sources, and the contingency of a one-line outage is considered. We discuss the most severe operating state, outage of the line between busbar 1 and busbar 5, for VAR support [9] The initial operating condition, one transmission line outage, of the system is unstable, and $S M=-17.8 \%$, which means the power flow equation cannot obtain a convergence solution in the initial state. In spite of the initial state being a nonfeasible configuration, the methodology can search for a feasible solution at the initialisation process.

A decision vector $y=[1.03,0.9,0.2]^{T}$ and the vector $\boldsymbol{w}=[0.4,0.3,0.3]^{T}$, the preference direction of the DM towards the goal, are given. The optimum solution is presented in Table 4 . The optimum value $\alpha$, which is a nega-

Table 4: Results of study 2 (AEP 14-busbar system)

\begin{tabular}{llllr}
\hline & $\begin{array}{l}\text { Contingency } \\
\text { condition }\end{array}$ & $\begin{array}{l}\text { IFS } \\
\text { solution }\end{array}$ & $\begin{array}{l}\text { DM } \\
\text { goals }\end{array}$ & $\begin{array}{l}\text { After } \\
\text { planning }\end{array}$ \\
\hline Loss $(100 \mathrm{MW})$ & $*$ & 0.6802 & - & 0.5002 \\
Cost $\left(10^{10} \$ \mathrm{NT}\right)$ & $*$ & 1.3766 & 1.03 & 1.0127 \\
$1-S M$ & 1.178 & 0.9990 & 0.90 & 0.8215 \\
$\sum_{\alpha} V D_{i}$ & $*$ & 1.5761 & 0.20 & 0.1120 \\
& $*$ & 4.5872 & - & -0.0434 \\
\hline $\boldsymbol{w}=[0.4,0.3,0.3]^{r}$ & & & \\
$*$ unstable state & & & \\
IFS = initial feasible state & & &
\end{tabular}

tive number, informs the DM that the decision strategy is attainable, and an improved solution is obtained. This study required $931 \mathrm{~s}$ total computer time on a SUN SPARC Station 2 to obtain the optimum solutions.

\section{Conclusions}

In this paper, a new formulation for the VAR source planning problem, treating it as a constrained, multiobjective, nondifferentiable optimisation problem, is presented. The feature of this formulation is that the objectives include the cost of system operation, investment cost of VAR sources, security margin of the system and voltage deviation of the system. They are mainly concerned with the VAR planning problem.

The goal-attainment based simulated annealing method for solving a general multiobjective optimisation problem with nonconvex function in objective space is developed. The authors believe that the solution scheme constitutes an effective approach for dealing with decision-making processes, which are particularly evident in multiobjective VAR planning problem cases. By the proposed method, the DM can find a desirable, global efficient solution for the multiobjective optimisation problem. The numerical results have proved the performance of the solution methodology. Hence, the algorithm is a powerful tool for planners to develop a correct investment policy in $\mathrm{MO}$ problems.

\section{References}

1 HAPP H.H and WIRGAU, KA : 'Static and dynamic VAR compensation in system planning', IEEE Trans., 1978, PAS-97, pp. $1564-1572$

2 FER NANDES, R.A., LANGE, F., BURCHETT, R.C., HAPP, H.H., and WIRGAU, K.A.: 'Large scale reactive power planning', IEEE Trans., 1983, PAS-102, pp. 1083-1088

3 KISHORE, A., and HILL, E.F.: 'Static optimization of reactive power sources by use of sensitivity parameters', IEEE Trans., 1971, PAS-90, pp. 1166-1173

4 IBA, K., SUZUKI, H., SUZUKI, K., and SUZUKI, K.: 'Practical reactive power allocation/operation planning using successive linear programming', IEE E Trans., 1988, PWRS-3, (2), pp. 558-566

5 SACHDEVA, S.S., and BILLINTON, R.: 'Optimum network VAR planning by nonlinear programming', IEEE Trans., 1973, PAS-92, pp. $1217-1225$

6 AOKI, K., FAN, M., and NISHIKORI, A.: 'Optimal VAR planning by approximation method for recursive mixed-integer linear programming', IEEE Trans., 1988, PWRS-3, (4), pp. 1741-1747

7 LEBOW, W.M., ROUHANI, R., NADIRA, R., USORO, P.B., MEHRA, R.K., SOBIESKI, D.W., PAL, M.K., and BHAVARAJU, M.P.: 'A hierarchical approach to VAR optimization in system planning', IEEE Trans., 1985, PAS-104, (8), pp. 2015-2057

8 OPOKU, G.: 'Optimal power system VAR planning', IEEE Trans., 1990 , PWRS-5, (1), pp. 53-59

9 OBADINA, O.O., and BERG, G.J.: 'VAR planning for power system security', IEEE Trans., 1989, PWRS-4, (2), pp. 677-686

10 HONG, Y.Y., SUN, D.I., LIN, S.Y., and LIN, C.J.: 'Multi-year multi-case optimal VAR planning', IEEE Trans., 1990, PWRS-5, (4), pp. $1292-1301$

11 OBADINA, O.O., and BERG, G.J.: 'Determination of voltage stability limit in multimachine power systems', IEEE Trans., 1988, PS-3, (4), pp. 1545-1554

$12 \mathrm{CHU}, \mathrm{K}-\mathrm{C}$.: 'On the noninferior set for the systems with vectorvalued objective function', IEEE Trans., 1970, AC-15, pp. 591-593

13 GEMBICKI, F.W., and HAIMES, Y.Y.: 'Approach to performance and sensitivity multiobjective optimization: the goal attainment method', IEEE Trans., 1975, AC-20, (6), pp. 769-771

14 CHANKONG, V., and HAIMES, Y.Y.: 'Multiobjective decision making: theory and methodology' (North-Holland, New York, 1983

15 AARTS, E., and KORST, J.: 'Simulated annealing and boitzmann machines' (John Wiley \& Sons Ltd., 1989)

16 KIRKPATRICK, S., GELATT, C.D., and VECCHI, M.P.: 'Optimization by simulated annealing', Science 220,1983 , pp. 671-679

17 OTTEN, R.H.J.M., and VAN GINNEKEN, L.P.P.P.: 'The annealing algorithm' (Kluwer Academic Publishers, 1989)

18 LUNDY, M., and MEES, A.: 'Convergence of an annealing algorithm' Math. Program., 1986, 34, pp. 111-124

19 ROMEO, F., and VINCENTELLI, S.: 'Probabilistic hill climbing algorithms: properties and applications'. Proc. Chapel Hill Conf. on VLSI, 1985, Chapel Hill, pp. 393-417

20 CHIANG, H.D., WANG, J.C., COCKINGS, O.R., and SHIN, H.D.: 'Optimal capacitor placements in distribution systems: Part 1: A new formulation and the overall problem', IEEE Trans., 1990. PWRS-5, (2), pp. 634-642

21 CHIANG, HD WANG, JC, COCKINGS, OR and SHIN, H.D.: 'Optimal capacitor placements in distribution systems: Part 2: Solution algorithms and numerical results', IEEE Trans., 1990, PWRS-5, (2), pp. 643-649

22 HSIAO, Y.T., LIU, C.C., CHIANG, H.D., and CHEN, Y.L.: 'A new approach for optimal VAR sources planning in large electric power systems'. IEEE Summer Power Meeting, paper 92 SM440-8 PWRS, Seattle, WA, July 12-16, 1992 